\title{
La storiografia medica nell'età della Rivoluzione: l'esperienza francese e italiana
}

\author{
di Luciano Bonuzzi
}

\section{Riassunto}

Dopo un breve cenno alla storia della storiografia medica da Ippocrate all'età della grande Rivoluzione, si confrontono $i$ contributi italiani e francesi editi alla fine del'700

Nel '700 la storia della medicina segue indirizzi annalistici, bibliografici e metodologici. In Francia, con Pinel e Cabanis, la storia della medicina ha soprattutto carattere metodologico come aveva insegnato Cullen. Anche in Italia, dove l'interesse per la disciplina è più tardivo rispetto agli altri paesi europei, si impone il modello metodologico trasmesso dalla cultura di lingua francese.

Quando si riflette sui problemi della medicina nell'età della grande Rivoluzione il pensiero corre immediatamente al dibattito sugli ospedali, alla nascita dell'igiene pubblica, alla strutturazione specialistica del sapere. E pur tuttavia è la discussione, assolutamente nuova, sul senso della storiografia medica che dà la misura più fedele di quanto stia cambiando la cultura medica tradizionale. Ed ancora una volta si avverte con chiarezza come la quotidianità del malato, dall'organizzazione degli ospedali agli indirizzi terapeutici, sia strettamente legata ai grandi mutamenti ideologici.

L'incontro fra medicina e storia - la questione che ci si vuole mettere a fuoco - avviene con l'Autore ippocratico in un momento fra i più stimolanti della tradizione occidentale quando, nell'universo del male che sostanzia la condizione umana, affiora il concetto scientifico di malattia che viene interpretata in chiave umorale. Mentre in Natura dell'uomo l'Autore ippocratico propone lo schema antropologico umorale destinato a durare fino alla scoperta della circolazione del sangue, in Antica medicina lo stesso Ippocrate elabora infatti il primo modello laico di storiografia medica. Gli è che la cura delle malattie, che vanno osservate tenendo conto dell'ambiente naturale, diventa possibile grazie al progresso della dietetica, con il passaggio dal crudo al cotto e con la diluizione del cibo ${ }^{1}$. L'asse portante della storia della 
medicina coincide insomma con la storia della dietetica che, d'accordo con Democrito $^{2}$, si configura come un momento essenziale per il progresso dell'umanità.

Se con Ippocrate affiora la proposta affascinante di una storia antropologica della medicina quasi per seguirne e controllarne le possibilità concrete, con Galeno invece si configurano i nuovi problemi storiografici maturati all'interno della medicina stessa. Galeno affronta infatti la questione della correttezza bibliografica ${ }^{3}$, ma confronta anche i vari indirizzi metodologici che orientano le differenti scuole ${ }^{4}$.

Con Plinio si impone poi, un secolo prima di Galeno ed in termini ormai maturi, per quanto velenosamente polemici, la riflessione sulla posizione sociale del medico e sui limiti della medicina, ritenuta un'arte da avventurieri greci che Plinio contesta ${ }^{5}$.

Nel mondo antico, la riflessione storica sulla medicina rivela una grande complessità ed apertura culturale. Ma nell'età di mezzo, mentre all'interesse per la salute subentra l'ansia per la salvezza, la curiosità critica che spinge alla verifica storica decade proprio come decade la ricerca biologica che resta sostanzialmente ferma ai libri di Galeno e di Plinio.

Il bisogno di storia, se si passa l'espressione, ricompare in medicina fra il '400 e il ' 500 con Tortelli e Bartolotti ${ }^{6}$. Si deve a Tortelli una cronaca sulle origini mitologiche della medicina e sullo stato dell'arte in epoca grecoromana. Bartolotti è invece più attento alla possibilità di distinguere i vari indirizzi medici in base alle categorie ideologiche che sottendono sia la costruzione del sapere che l'elaborazione delle tecniche terapeutiche; parla infatti di medicina magica, sacerdotale etc.

Ma la storia della medicina si propone all'attenzione degli studiosi in modo inequivocabile solo con il classico trattato di Daniel Le Clerc ${ }^{7}$, edito molti anni dopo la rinascita dell'anatomia e un secolo dopo la scoperta della circolazione del sangue. La riflessione storica, in effetti, rivela la propria incisività in un momento critico quanto mai per l'evoluzione del sapere medico, proprio quando in seguito alla scoperta della circolazione del sangue risulta falsificata la millenaria antropologia umorale e si impongono nuovi modelli antropologici ad impronta meccanicista.

Lungo il '700 comunque si delineano tre fondamentali indirizzi nella ricerca storico-medica: ad impronta annalistica, bibliografica e metodologica. Ma un cenno spetta anche al dizionario dell'Eloy ${ }^{8}$ elaborato, alla maniera del dizionario del Bayle, elencando in ordine alfabetico i medici più famosi ed illustrandone la biografia e le tecniche ritenute degne di menzione. 
L'approccio annalistico esordito con Le Clerc raggiunge il livello più maturo con la Storia prammatica della medicina di Curzio Sprengel che propone un'esposizione cronologica «della cognizione dottrinale delle malattie comuni alla specie umana, del loro trattamento e delle mutazioni sì della teoria che della pratica medica». La storia della medicina secondo Sprengel deve poi essere «realmente prammatica» nel senso di Plutarco che parla di storia prammatica per significare una storia che tende direttamente ad un'utile istruzione ${ }^{9}$.

Fra le opere ad indirizzo bibliografico si ricordano invece le monumentali rassegne di quel grande umanista e scienziato che fu Haller: la Bibliotheca anatomica (Tiguri 1774-77), la Bibliotheca botanica (Tiguri 1771-72), la Bibliotheca chirurgica (Bernae et Basileae 1774-75) e la Bibliotheca medicinae practicae (Basileae et Bernae 1776-88). Ed ancora va ricordato il commento di van Swieten ${ }^{10}$ agli aforismi di Boerhaave; un commento che fornisce una esauriente bibliografia ragionata di tutto il sapere accumulato dalla medicina fino alla metà del '700.

È palese in questi studi la preoccupazione di fornire, sia pure in un contesto storico, il più ampio numero di nozioni che presentino immediata utilità. Tale orientamento rimanda alla consuetudine secolare di citare gli antichi quando l'umoralismo, prima della scoperta della circolazione del sangue, garantiva una sostanziale continuità del sapere.

Abbastanza diverso è l'intento di quegli studiosi che si rivolgono alla storia con preoccupazioni di ordine essenzialmente metodologico come fanno Cullen o Pinel.

Per Cullen la storia è un campo di verifica. Di fronte alla fondamentale questione se l'esercizio pratico della medicina debba poggiare sul ragionamento o piuttosto restare fermo alla mera esperienza, si orienta infatti ricorrendo alla comparazione storica e prende atto che la pratica clinica ha sempre fatto riferimento a principi stabiliti con il ragionamento, come dimostra la successione dei sistemi medici. Per suo conto guarda soprattutto a Bacone e a Galilei, ma non si tratta di un ingenuo induttivista collocabile fra quanti credono di poter collaborare alla costruzione di un sapere staticamente ordinabile. Ed è ancora la prospettiva storica dei problemi che lo invita ad accettare la provvisorietà come connaturata al sapere che ritiene suscettibile di revisione continua. Ogni nuova scoperta, come osserva com- 
mentando Boerhaave, impone infatti la necessità di rinnovare il precedente sistema medico per sostituirlo con un modello che sia in grado di integrare le nuove osservazioni ${ }^{11}$.

Con Pinel, che pubblica le proprie opere più significative nell'anno VI e IX della nuova era inaugurata dalla grande Rivoluzione (1797/98 e 1800/ 1801), la compromissione teoretica con la storia diventa ancora più forte ${ }^{12}$. Pinel del resto traduce in francese ${ }^{13}$ le opere di Cullen e di Baglivi, un ippocratico che sostiene con passione la medicina d'osservazione.

Per Pinel il mondo della storia è fatto di luci e di ombre d'oscurantismo che possono ostacolare la marcia trionfale e progressiva dei lumi che in medicina si accendono dove brilla lo spirito d'osservazione. Da questo presupposto deriva l'entusiasmo per la medicina ippocratica, ritenuta medicina d'osservazione per antonomasia, in antitesi con l'umoralismo galenico che, per Pinel, è intriso di sottigliezze speculative.

Dato che Pinel intende guardare alla sola medicina d'osservazione, sorvola disinvoltamente sull' opportunità della completezza documentaria. Le rassegne bibliografiche agli occhi di Pinel sembrano semplici dizionari; van Swieten, in particolare, con il magistrale commento a Boerhaave è ritenuto prolisso.

Tale atteggiamento riflette un po' il bisogno illuminista di semplicità e di chiarezza che tende a scotomizzare quanto si rivela problematico e complesso.

A Pinel interessa la sola medicina d'osservazione che esordisce in Grecia e riconosce in Ippocrate l'ideale maestro. Pinel peraltro sa bene che un accostamento alla medicina ippocratica non può più essere diretto, ma mediato in chiave metodologica. Il rimando ad Ippocrate del resto - dopo Bacone, Cullen e Baglivi - è un pò una bandiera per tutti gli studiosi settecenteschi che valorizzano l'osservazione e che attribuiscono alla medicina una funzione promozionale per il progresso dell'uomo e per la liberazione dalla malattia : una concezione tipicamente ippocratica, in antitesi con il punto di vista di Rousseau che riconosce nello stato di natura il momento mitico della salute che viene guastata dal progresso.

L'entusiasmo ippocratico non è spiegabile con rimandi ad esclusive opere di medicina. L'ammirazione per la Grecia, per Ippocrate e per Plutarco, che per Pinel è insieme a Tacito il modello dei biografi, va infatti inserita nel culto dell'antichità classica che caratterizza l'età di Pinel in ogni manifestazione culturale. Nella seconda metà del ' 700 in effetti si va sempre più diffondendo il gusto neoclassico e, come nota Starobinski ${ }^{14}$, il «ritorno all' antico» pre- 
cede ampiamente l'età della Rivoluzione. La lettura di Plutarco ha poi indiscussa fortuna dal Rinascimento al primo ' 800 .

In Pinel il consenso per l'ippocratismo si accompagna al rifiuto radicale di quanto rimanda al pensiero galenico. Tale dissenso dall'umoralismo galenico, scontato dopo la scoperta della circolazione del sangue, non ha più il mordente dell'età che ha visto la rinascita dell'anatomia. Anche la lotta all'umoralismo acquista però qualche interesse sul piano linguistico esprimendo a questo livello un preciso bisogno tecnico-culturale ma anche sociale. In osservanza all'insegnamento di Locke e di Condillac, per Pinel è indispensabile disporre di un apparato linguistico semplice e preciso per superare le incertezze immanenti alla medicina umorale. Nel contempo la precisione scientifica del linguaggio gli permette di prendere le distanze dalla medicina popolare che si esprime, pure questa, con linguaggio umorale. Ad un simile bisogno di rinnovamento linguistico, ormai improrogabile in medicina, non è forse estranea la tendenza dell'illuminismo ad allontanarsi da una partecipazione autentica alla cultura e al linguaggio popolare così come tende ad allontanarsi dai modi dell' Ancien Régime quando, ancora alla vigilia della Rivoluzione, la sfumatura del linguaggio era considerata negli ambienti aristocratici come un tratto fondamentale delle buone maniere.

In sintesi il disegno storico-medico di Pinel ruota intorno alle emblematiche figure di Galeno e di Ippocrate che, secondo una congettura di vago sapore manicheo, incarnano rispettivamente la sottigliezza speculativa e lo spirito d'osservazione. In altre parole, l'utilità pratica della storia deriva dalla possibilità di ricomporre le vicende della medicina d'osservazione per fondare una corretta metodologia clinica che esige strumenti adeguati che vanno dalla precisione dell'apparato linguistico al rigore nella ricerca quale permette il solo ambiente ospedaliero. Il costante riferimento al pensiero ippocratico, che è il filo conduttore della storia della medicina, comporta poi la valorizzazione del naturalismo, della dietetica e della concezione biografica della malattia che Pinel trova riproposta da Cullen puntualmente attento a tutti i fattori che permettono di ricostruire la vicenda individuale del malato.

Pinel con le sue preoccupazioni metodologiche è assai vicino a Cabanis, il più illustre ideologo della medicina che opera in Francia nell'età della Rivoluzione. Cabanis in anni di profondi rivolgimenti culturali ed amministrativi affronta infatti con passione, proprio come Pinel, la complessa questione dell'assistenza sanitaria ed ospedaliera e si pone nel contempo il problema antropologico. Gli è che l'immagine seicentesca dell'uomo centrata 
sulla pompa idraulica del cuore, dopo le ricerche di Haller sul sistema nervoso, è mutata ed è al centro di un acceso dibattito che investe il rapporto fra il fisico e il morale. Immagine dell'uomo e qualità dell'assistenza sono insomma problemi, teorici e pratici, che mettono in discussione il senso stesso della medicina che Cabanis analizza nel Coup d'œil sur les révolutions et sur la réforme de la médecine ${ }^{15}$, un 'opera che forse troppo severamente Moravia giudica «disordinata e farraginosa». Lo stesso Moravia, d'altra parte, sottolinea il tono «militante» di questo saggio che auspica «una medicina consapevole della propria vocazione antropologica ${ }^{16}$.

Cabanis nella sua revisione storica ribadisce - alla maniera di Cullen e di Pinel - l'importanza fondamentale del metodo nelle scienze d'osservazione; riserva all'errore un'attenzione privilegiata in quanto è proprio l'errore che impone una continua revisione del sapere; contro le seduzioni ingannevoli del ragionamento deduttivo e contro l'imprecisione semantica raccomanda la precisione del linguaggio ed invita alla semplificazione del sapere per cogliere con evidenza i rapporti che legano le varie scienze fra loro; individua nel modello terapeutico la chiave di volta che scandisce l'evoluzione del pensiero medico; riconosce infine che l'esercizio della medicina è strettamente legato al contesto sociale e politico. Indubbio l'entusiasmo per Ippocrate che «liberò la medicina dai falsi sistemi e la fornì di metodi sicuri» rendendo più ricca, grazie ai lumi della medicina, la stessa filosofia. In Ippocrate infatti, nota Cabanis, l'osservazione guida il ragionamento con sicurezza e con rigore. I momenti fortunati della storia della medicina coincidono infatti, secondo Cabanis, con la riscoperta dell'ippocratismo ${ }^{17}$.

Ed ancora - argomenta Cabanis - la stessa costruzione e trasmissione del sapere deve tener conto della concreta organizzazione biologica dell'uomo. Fra gli antichi, Ippocrate Aristotele ed Epicuro hanno affrontato questo problema ma solo dopo Bacone, con Locke etc., è stata possibile una «conoscenza più esatta delle facoltà e delle operazioni dello spirito umano» ${ }^{18}$. In altre parole è solo in prospettiva sensista che viene affrontata correttamente la questione della fondazione e della trasmissione del sapere.

Per la corretta trasmissione del sapere è in ogni caso indispensabile un linguaggio adeguato; ed è soprattutto il linguaggio scritto, quando è preciso, che favorisce la rapidità del progresso. Ma troppo spesso il linguaggio della medicina per adeguarsi ai pregiudizi popolari si rivela impertinente. Gli stessi insegnanti francesi di medicina, vincolati alla terminologia dei Greci e degli Arabi, non di rado si sono limitati a francesizzare parole sempre più estrane e pertanto hanno scritto con stile oscuro e triviale ${ }^{19}$. È invece 
opportuna la conoscenza diretta delle lingue classiche e di quelle moderne perchè le traduzioni appiattiscono il pensiero e possono essere infedeli.

Cabanis, che è profondamente consapevole di scrivere ed operare in un momento irrepetibile ${ }^{20}$, si rivolge insomma alla storia per cogliere gli strumenti che rendono possibile il progresso dell'uomo e la sua liberazione dalla malattia: pilastri di tale lotta, come dimostra la storia, sono la consapevolezza antropologica, la precisione del linguaggio, il rigore metodologico. Cabanis in effetti con il suo impegno prammatico è vicino, ancor più di Pinel, allo spirito di Condorcet ${ }^{21}$ che intende la storia come una marcia felice del progresso delle scienze.

L'impegno alto, in senso metodologico e politico, della storiografia medica francese elaborata nell'età della Rivoluzione non trova analogo, contemporaneo riscontro negli altri paesi europei. Si pensi a Sprengel che, pur attento agli aspetti «prammatici» del suo lavoro, mantiene sempre il distacco dell'erudito.

Per quanto poi riguarda la storia della medicina in Italia, così come si è sviluppata lungo il ' 700 , si nota forse una vitalità ancora più modesta rispetto a quella affiorata negli altri paesi. Anche nell'Italia settecentesca tuttavia non mancano i ricercatori dalla forte passione umanistica ma la citazione storico-medica è per lo più concepita in chiave di mera allusione bibliografica. La modesta sensibilità storica contrasta poi con la grande attenzione portata al problema metodologico dagli studiosi più illuminati. Si pensi a Baglivio allo stesso Morgagni.

Alla fine del ' 600 , mentre il mondo scientifico è ancora stupito per la scoperta della circolazione del sangue che ha falsificato la millenaria antropologia umorale, Baglivi ${ }^{22}$ raccomanda la «massima necessità delle osservazioni in fatto di medicina»: Bacone gli fornisce la guida teoretica, Galilei il modello sperimentale. Nel contempo Ippocrate è considerato il «primo fra i medici» in quanto «non parla come uomo ma colla voce della natura». L'entusiasmo ippocratico e la consapevolezza che la medicina è «figlia del tempo» non stimolano tuttavia un'autentica ricerca storica. Baglivi, d'altra parte, diffida dei libri che per lo storico costituiscono invece uno strumento di lavoro essenziale. I libri - insiste Baglivi - vanno accostati con prudenza, con sobrietà, con preoccupazione per gli errori che possono contenere senza farsi prendere la mano dall'entusiasmo per gli antichi e senza scordare i rischi 
immanenti al sapere astratto. Il vero libro è il malato. Dietro questa posizione di Baglivi, accanto all'entusiasmo per il metodo sperimentale, si coglie l'influenza impalpabile di suggestioni complesse maturate al di fuori della medicina, come la perplessità controriformista nei confronti dei «cattivi libri» e la valorizzazione tradizionale della comunicazione orale apprezzata, ancora una volta, da Muratori ${ }^{23}$ verso la metà del secolo.

Anche in Morgagni, il patologo più illustre del ' 700 , la storia della medicina ha uno scarso rilievo che, nell'insieme degli scritti, appare come una zona d'ombra in contrasto con la grande sensibilità culturale testimoniata dal rigore metodologico, dalle preoccupazioni didattiche e dall'ampiezza degli interessi umanistici che spingono il maestro padovano ad occuparsi, con autentica competenza, di storiografia generale come rivelano le Epistole Emiliane. In medicina invece la ricerca storica non va oltre la preoccupazione per la completezza bibliografica.

In effetti, la crescita della storiografia medica nella cultura italiana del '700 appare abbastanza difficile ed è forse scoraggiata da una sensibilità generale poco attenta alla relatività del sapere scientifico, fiduciosa piuttosto sulle certezze «astoriche» di un mal inteso spirito galileiano. Una significativa eccezione è però rappresentata dalla «Storia delle Scoperte FisicoMedico-Anatomico-Chirurgiche fatte dagli uomini illustri italiani» ${ }^{24}$ di Brambilla che muove nell'atmosfera culturale austriaca. Il Brambilla pur riconoscendo, nella Prefazione al trattato in parola, che gli uomini d'ingegno sono cittadini del mondo non disdegna infatti di occuparsi degli «Illustri Italiani».

Verso la metà del ' 700, ad ogni modo, anche la cultura medica italiana dà segni di rinnovamento: escono a Napoli in edizione italiana sia il Dizionario dell'Eloy (1761) che la Storia della Medicina di Le Clerc (1762) che verosimilmente trovano un pubblico stimolato da interessi storici e critici più forti che nel passato, dato il momento profondamente inquieto che, ancora una volta, attraversa la medicina moderna. Mentre le ricerche di Haller propongono un nuovo modello antropologico centrato sul cervello, l'umoralismo infatti è decisamente finito. In questo contesto si impone l'esigenza di aggiornare il metodo con la guida ormai consolidata di Bacone e di Locke. Nel contempo il linguaggio impertinente dell'umoralismo tende ad essere sostituito da quello proposto dalla chimica mentre al latino subentra il volgare. Si impone poi il naturalismo e, ancora una volta, si cercano in Ippocrate le radici da cui muove il richiamo alla natura e all'ambiente. In un momento tanto complesso l'interesse per l'analisi dello sviluppo del sapere 
diventa più forte che in passato e favorisce, fatalmente, la ricerca storicomedica.

Il naturalismo, motivo centrale dell'ippocratismo, promuove allora l'interesse per l'ambiente e per la possibilità delle terapie fisiche. Le cure termali, in particolare, vivono un nuovo rilancio che fa leva, come nell'opera di Mandruzzato ${ }^{25}$ dedicata ad Abano, sulla lunga storia di una felice prassi terapeutica che può ormai essere riconsiderata con il moderno linguaggio della chimica.

Anche la riflessione storica sul metodo si impone finalmente nella cultura medica italiana dell'ultimo '700. Comparetti, un maestro dello Studio di Padova come Mandruzzato, pubblica nel 1793 un interessante Saggio della scuola clinica nello spedale di Padova. Il saggio in parola esordisce sottolineando il contrasto fra la condizione del malato esposto nelle piazze dell'antica Babilonia e le possibilità di approccio oggettivo e scientifico fornite dal solo ospedale. Comparetti, imbevuto di spirito baconiano, segue le fortune del metodo d'osservazione e ricorda ancora una volta che l'osservazione analitica esordisce in medicina con Ippocrate ma aggiunge che nella seconda metà del '500, poprio nell'ospedale padovano di S. Francesco, Albertino Bottoni e Marco Oddo, dietro la richiesta della Nazione Alemanna, iniziano l'insegnamento clinico al letto del malato.

L'interesse per la storia della didattica ospedaliera muove, nel pensiero di Comparetti, dalla considerazione che l'insegnamento clinico «non può essere collocato, se non negli Spedali, dovendosi istruire gli allievi più pegli occhi, che pegli orecchi». Il problema della didattica in ambiente ospedaliero affiora anche in Francia, lungo gli anni '70 e '80, in margine agli sforzi per riordinare l'Hôtel-Dieu. Nell'occasione viene anche bandito l'invito a «determinare qual sia il miglior modo d'insegnare la medicina pratica in uno Spedale», incoraggiando a risolvere la questione proprio «chi spezialmente ha insegnato, o studiato attualmente la Clinica in qualche Università» ${ }^{26}$. Stimolato da questo tema, Comparetti analizza criticamente $i$ più celebri ospedali e traccia il progetto di un ospedale ideale muovendo dalle indicazioni ambientali puntualizzate da Ippocrate, considerando il rapporto fra ampiezza dello stabilimento e popolazione servita, discutendo ogni momento della vita ospedaliera e proponendo un «Foglio di visita» e un «Foglio di osservazione» che permettano di registrare per iscritto, in forma standardizzata, i principali segni clinici e provvedimenti terapeutici. Profondamente attento ai fermenti della cultura europea, e segnatamente francese, Comparetti traccia insomma una storia dell'osservazione e della didattica 
clinica mettendo a fuoco la scrittura standardizzata della cartella clinica. È così in sintonia con lo spirito d'ordine legato alla scrittura, tipico dell'Encyclopédie.

Con Comparetti hanno ormai preso corpo anche in Italia le prospettive storico-mediche dell'illuminismo, ma solo con Scuderi $^{27}$, che divide per epoche la medicina antica e quella moderna, si ha un vero tentativo di guardare alla storia della medicina in senso globale. Scuderi, fra l'altro, sottolinea con lucidità l'importanza dottrinale e non solo clinica dell'opera di Cullen. Del resto nel 1796, mentre già incombeva l'occupazione militare francese, era uscita a Venezia la traduzione degli Elementi di medicina pratica di Cullen che contiene, come si è ricordato, una breve ma problematica puntualizzazione storica sulle più recenti scuole di medicina.

Nell'ultimo decennio del '700 la storiografia ad impronta metodologica, di ispirazione illuminista, ha insomma fatto breccia nella cultura medica italiana. Ma dopo l'occupazione francese gli interessi storico-critici diventano ancora più forti soprattutto fra Padova e Pavia.

Indubbiamente originali sono i contributi storici di Rasori. Il cittadino Rasori nel Discorso recitato nell'assumere la Cattedra di Clinica in Pavia il giorno 10 Frigerio, anno VIII (27 novembre 1798) contesta il «preteso genio d'Ippocrate», deformato dal linguaggio e dai pregiudizi dei commentatori : è ormai lo spirito di polemica che investe se stesso. Più interessante e più pacata è invece la prolusione Sul metodo degli studi medici del 1809 dove valorizza l'opera del Montano, l'illustre Maestro padovano che ancor prima di Bottoni e di Oddo ha inaugurato l'insegnamento clinico in ospedale al letto del malato. Scrive infatti Rasori:

«Sono gli Spedali il grande e solo tempio sacro alla scienza e alla istruzione medica, le quali hanno ivi sede perenne» ${ }^{28}$.

Accanto all'interesse per l'osservazione ospedaliera affiora in questo saggio una certa fierezza nazionalistica che è un elemento abbastanza nuovo ed estraneo alla cultura cosmopolita del secolo precedente.

Anche a Padova, negli anni francesi, la storia della medicina rifiorisce come a Pavia. A Padova dopo la morte di Morgagni l'atmosfera della facoltà medica cambia rapidamente. La neurofisiologia di Haller è ben presto condivisa da molti studiosi. E Gallini, un fisiologo a formazione europea, sintetizzando i motivi essenzali della cultura d'oltralpe divulga dalla cattedra un modello antropologico di ispirazione sensista centrato sul sistema nervoso $^{29}$. Dopo anni ricchi di fermenti, nel 1815 vienne addirittura istituita 
una Cattedra di Storia e Letteratura Medica che, occupata del Montesanto ${ }^{30}$, sarà però disattivata dopo un paio d'anni dagli Austriaci, forse perchè era ritenuta un focolaio di nazionalismo.

Montesanto non si occupa di sola storia ma anche nelle richerche di patologia non trascura il rimando agli autori del passato e sottolinea l'importanza che spetta al rapporto fra lo stato di salute e le condizioni economiche e sociali dei pazienti. Più originali sono comunque i contributi ad indirizzo esplicitamente storico. Le Memorie storico-critiche elaborate Intorno alle antiche dottrine italiane sulla contagione ${ }^{31}$ sono un'occasione per ricordare gli studiosi italiani ed in particolare Fracastoro. Nella monografia sull'Origine della clinica medica etc. ${ }^{32}$ torna invece sulla priorità padovana dell'insegnamento clinico in ospedale ricordando come Marco Oddo avesse migliorato la didattica accompagnando gli allievi ad Abano per visitare i pazienti inviati alle terme. Con Montesanto, mentre la storia acquista accenti nazionalistici e rivela indubbie aperture sociali, il controllo dei documenti è ormai rigoroso.

Il magistero di Montesanto si prolunga idealmente in Cervetto ${ }^{33}$ che nel 1860 inaugura l'insegnamento di Storia della Medicina a Bologna. Secondo Cervetto la storia della medicina, che ha compiti di verifica metodologica e di riflessione critica sugli aspetti civili che condizionano la salute, va concretamente elaborata attraverso l'analisi biografica dei medici più rappresentativi. La biografia illustre è ritenuta infatti uno stimolo ed un credibile modello per i giovani; la biografia, oltretutto, permette di valorizzare tante priorità italiane, fin troppo trascurate. L'impegno politico e l'attenzione scrupolosa alle fonti sono i tratti che caratterizzano l'opera di Cervetto.

Nell' 800 , ad ogni modo, la storiografia medica italiana vive il momento più felice con De Renzi e soprattutto con Puccinotti. Si tratta peraltro di una storiografia complessa che solo in parte rimanda a suggestioni di origine francese. Puccinotti, in particolare, è assai sensibile al modello elaborato da Sprengel ed agli inviti della filosofia tedesca. 


\section{Note e richiami bibliografici}

1 Ippocrate, L'antica medicina, 3-5, in Opere, a cura di M. Vegetti, Torino 1976, 164-167.

2 Democrito, in O.p. cit. alla nota 1, 146-147.

3 Galeno, I miei libri, in Opere scelte, a cura di I. Garofalo e M. Vegetti, Torino 1978, 61 e seg.

4 Galeno, Le scuole di medicina, in Op. cit. alla nota 3, 103 e seg.

5 Plinio, Storia Naturale, XXIX, 8.

6 Tortelli, G., De medicina et medicis; Bartolotti, G., De antiquitate medicinae, a cura di D. M. Schullian, L. Belloni, Milano 1954.

7 Le Clerc, D., Histoire de la médecine, La Haye 1729.

8 Eloy, N., Dictionnaire historique de la médecine etc., Mons 1778. La prima edizione è del 1755.

9 Sprengel, C., Storia prammatica della medicina, ed. it., Napoli 1824, 1-3.

10 van Swieten, G., Commentaria in Hermanni Boerhaave etc., Lugduni Batavorum, 174276.

11 Le riflessioni di W. Cullen intorno alla storia del metodo in medicina sono espresse nell'ampia Prefazione che precede gli Elementi di medicina pratica (ed. it., Venezia 1796).

12 Per una sintesi del pensiero storico-medico di Pinel, si veda: Nosographie philosophique ou la méthode de l'analyse appliquée à la médecine, Paris an VI, I-XXXIX; Traité médicophilosophique sur l'aliénation mentale ou la manie, Paris an IX, I-LVI.

13 Su Pinel filologo si veda: C. Deulin, Pinel, homme de lettres, Ann. Méd.-Psychol., 12 série, II (1927), 52.

14 Starobinski J., 1789 et les emblèmes de la raison, Milano 1973, 5, 130.

15 Cabanis, P. J. G., Coup d'œil sur les révolutions et sur la réforme de la médecine, Paris 1804.

16 Moravia, S., Introduzione, in: Cabanis, Rapporti tra il fisico e il morale dell'uomo, Bari 1972, XV.

17 Op. cit. alla nota $15,76-80$.

18 Ib., 193.

19 Ib., 219-221.

20 Ib., 436.

21 Condorcet, Abbozzo di un quadro storico dei progressi dello spirito umano, a cura di M. Minerbi, Torino 1969.

22 Baglivi, G., Opere complete, a cura di R. Pellegrini, Firenze 1842, 45-46, 66.

23 Muratori, L. A., De i pregi dell'eloquenza popolare, Venezia 1750.

24 Brambilla, A., Storia delle Scoperte Fisico-Medico-Anatomico-Chirurgiche fatte dagli uomini illustri italiani, Milano 1780-82.

25 Salvatore Mandruzzato è stato valorizzato di recente da L. Premuda in più contributi (Salvatore Mandruzzato - 1758/1837 - professore «Ad Thermas Aponenses», in: Zusammenhang, Festschrift für Marielene Putscher, Köln 1984, II, 681; La medicina, in: Storia della cultura veneta - Il settecento, Vicenza 1986, 5/II, 261). Il notro trattato Dei bagni di Abano (Padova 1789-93) è preceduto da un'ampia cronaca delle alterne fortune di queste terme. Fra tante citazioni, che testimoniano gli interessi storici di Mandruzzato, si ricorda volentieri un aneddoto dal sapore veneto-bizantino che sembra fiorito su qualche isola dell'Egeo: si allude alla scoperta di una miracolosa sorgente che sarebbe stata accompagnata dal ritrovamento di «un quadro dipinto con l'immagine di Maria» (II, 7). La 
sensibilità veneta, insomma, passa sempre per Bisanzio anche quando intende ricollegarsi al naturalismo antico.

26 Comparetti, A., Saggio della scuola clinica nello spedale di Padova, Padova 1793, 7, 12.

27 Scuderi, R., Introduzione alla storia della medicina antica e moderna, Napoli 1814.

28 Rasori, G., Sul metodo degli studi medici, Milano 1809, 47.

29 Gallini, S., Sull'utilità ed importanza delle molteplici occupazioni e dei molteplici esperimenti che costituiscono il presente metodo d'educazione, Padova 1813.

30 Rippa Bonati, M., L'opera storico-medica di Giuseppe Montesanto (1779-1839), in: La Storiografia Medica in Italia tra 1800 e 1950 : uomini e idee, Padova 1985, 211.

31 Montesanto, G., Intorno alle antiche dottrine italiane sulla contagione, Padova 1936.

32 Montesanto, G., Dell'origine della clinica medica in Padova, Padova 1827.

33 Gelmetti, P., Giuseppe Cervetto ed il suo insegnamento storico-medico a Bologna, in: Atti VII Biennale della Marca e dello Studio Firmano, Fermo 1967.

\section{Zusammenfassung}

\section{Die Geschichtsschreibung der Medizin im Zeitalter der Revolution: französische und italienische Erfahrungen}

Nach einem kurzen Streifzug durch die Geschichte der Historiographie der Medizin von Hippokrates bis zur Zeit der grossen Revolution werden die Ende des 18. Jahrhunderts erschienenen italienischen und französischen Beiträge einander gegenübergestellt. Im 18. Jahrhundert verfolgt die Geschichte der Medizin annalistische, bibliographische und methodologische Richtungen. In Frankreich wird die Historiographie von Pinel und Cabanis, entsprechend der Lehre Cullens, vor allem auf methodologischem Wege betrieben. Auch in Italien, wo das Interesse für diese Disziplin gegenüber anderen europäischen Ländern später erwacht, übernimmt man das methodologische Modell der Kultur französischer Sprache. 


\section{Résumé}

\section{L'historiographie médicale à l'époque de la Révolution: l'expérience française et italienne}

Après un bref survol de l'historiographie médicale depuis Hippoerate jusqu'à la Révolution, comparaison est faite entre les ouvrages italiens et français parus à la fin du $18^{\mathrm{e}}$ siècle.

Au 18 siècle, l'histoire de la médecine est essentiellement annalistique, bibliographique et méthodologique. En France, avec Pinel et Cabanis, cette histoire, suivant l'enseignement de Cullen, a surtout un caractère méthodologique. En Italie, où l'intérêt pour la discipline se manifeste plus tardivement que dans les autres pays européens, c'est le modèle méthodologique transmis par la culture française qui s'impose.

\section{Summary}

\section{Medical historiography in the age of the Revolution: the French and the Italian experience}

After a brief hint at the history of medical historiography from Hippocrates to the age of the French Revolution, the author compares the Italian and French contributions published at the end of the $\mathrm{XVIII}^{\text {th }}$ century. In that century, history of medicine shows annalistical, bibliographical and methodological trends. In France, with Pinel and Cabanis, it has a predominantly methodological character, as thought by Cullen. Also in Italy, where the interest for this discipline arises somewhat later than in other European countries, the French methodological model is accepted.

Dott. Prof. Luciano Bonuzzi

Istituto di Storia della Medicina

dell'Università di Padova

via G. Falloppio, 50

I-35121 Padova 